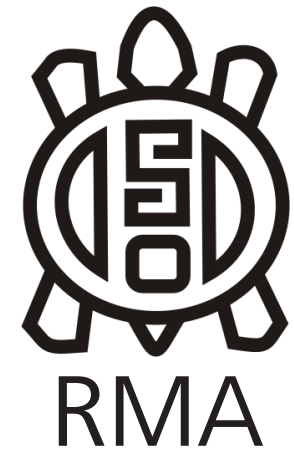

Arqueología

\title{
Identificando sitios arqueológicos del Período de Desarrollos Regionales (siglos X a XV d.C) en el bosque montano tucumano: la cuenca de Anfama (Dpto. Tafí Viejo)
}

\author{
Identification of Archaeological Sites from the Regional Development \\ Period (10th to 15th Centuries A.D.) in the Montane Forest of Tucuman: \\ Anfama Basin (Tafí Viejo) \\ Agustina Vázquez Fiorani*, Gonzalo Moyano** y Julián Salazar*** \\ *Becaria MA Archaeological Materials Science Program (ARCHMAT-Erasmus Mundus), \\ Email: agusfiorani@hotmail.com \\ ${ }^{* *}$ Centro de Investigaciones y Transferencia de Catamarca (CITCa), CONICET, \\ Email: gonzalexmoyano@gmail.com \\ *** Universidad Nacional de Córdoba, Instituto de Estudios Históricos, CONICET, \\ Email: juliansalazar@ffyh.unc.edu.ar
}

\begin{abstract}
Resumen
El objetivo de este trabajo es presentar los avances obtenidos en las tareas de identificación de sitios del Período de Desarrollos Regionales (siglos X a XV d.C) realizadas en la cuenca de Anfama (Dpto. Tafí Viejo, Tucumán), donde no se tenía ningún registro hasta el momento de ocupaciones asignables a este momento. Las actividades desarrolladas conjugaron tanto el trabajo con miembros de la comunidad indigena local como la intervención arqueológica, incluyendo la recolección de testimonios orales a los habitantes, el registro de piezas en su poder, prospecciones pedestres, relevamientos de brújula y cinta métrica corregidos a través de imágenes cenitales capturadas por drones y sondeos exploratorios y expeditivos. Consideramos que estos datos construidos mediante distintas metodologías constituyen un elemento para complejizar el debate sobre la naturaleza de los asentamientos de la primera mitad del segundo milenio de la Era en el piedemonte oriental de las Cumbres Calchaquíes.
\end{abstract}

Palabras clave: Período de Desarrollos Regionales; Prospección; Relevamiento; Sondeos exploratorios; Identificación

\begin{abstract}
The aim of this work is to present/report the advances made towards the identification of archaeological sites from the Regional Development Period (10th to 15th centuries A.D.) in the Anfama valley (Tafí Viejo, Tucumán), where no previous records of occupations during this period have been found. The activities developed combined both work with members of the community and archaeological intervention, including the collection of oral testimonies from the inhabitants, the record of pieces possessed by them, pedestrian surveys, compass and measuring tape mapping corrected through aerial images captured by drones, and exploratory surveys. We consider that these data gathered through different methodologies contribute to promoting the debate on the nature of the settlements of the first half of the second millennium A.D. on the eastern foothills of the Cumbres Calchaquíes.
\end{abstract}

Keywords:Late Intermediate Period; Pedestrian surveys; Mapping; Exploratory Surveys; identification.

\section{Introducción}

Como señalan Nielsen, Ávalos, y Menacho (2000) en el estudio de las sociedades agroalfareras del NOA la identificación de sitios ha estado fuertemente condicionada por el objetivo de reseñar sitios de gran tamaño, altamente concentrados y de fácil acceso, en desmedro de asentamientos más pequeños, estructuras, rasgos o artefactos aislados, así como la ausencia de todos ellos, considerados poco llamativos o difíciles de registrar o interpretar. Esta fisonomía disciplinar, impide una caracterización de la estructura del registro arqueológico y descarta información que puede resultar importante para comprender la organización espacial de las sociedades en el pasado (Nielsen et al. 2000) y no ha sido objeto de reflexiones sistemáticas. Sólo en algunos casos, estas problemáticas han sido trabajadas aunque fundamentalmente en contextos de cazadoresrecolectores (Scheinsohn 2001).

En esta línea, el Período de Desarrollos Regionales (siglos X a XV d.C, a partir de aquí PDR) ha sido tradicionalmente 
asociado a la existencia de centros poblados de arquitectura imponente, en su mayoría ubicados en altura y fácilmente reconocibles en el paisaje, como los que se registran en los valles intermontanos y en la Puna (Nastri 1999; Tarragó 1987, 1995). En el caso de las Cumbres Calchaquíes se ha tendido a dejar de lado asentamientos de menor escala, de carácter discreto y emplazados en áreas bajas, considerablemente más difíciles de identificar en el registro arqueológico. Solo recientemente nuevas investigaciones han situado el foco en espacios tardíos de dimensiones más reducidas, con el interés expreso de evaluar la transición del primer al segundo milenio de la Era (Palamarczuk 2016).

En el caso de la vertiente oriental de las Cumbres Calchaquíes, la baja visibilidad de los asentamientos asignables al PDR se ha equiparado a la virtual inexistencia de los mismos. Sin embargo, la baja representación de sitios del PDR con respecto a ocupaciones del Período Formativo no necesariamente responde a la ausencia de las primeras, sino también a divergencias en la intensidad, escala y duración de los asentamientos, a la visibilidad definida por las características propias de las dinámicas culturales y naturales de formación de los sitios, e incluso a los esfuerzos científicos orientados a identificarlos, variables analíticas de interés que no han sido abordadas en profundidad hasta el momento.

La identificación de nuevos sitios del PDR en los faldeos orientales, sin componentes arquitectónicos reconocibles o que no forman parte de las áreas cercanas a los grandes sitios ha quedado signada en muchos casos, al descubrimiento "accidental" o a acciones azarosas por parte de otros actores sociales, como grandes obras de construcción privadas y estatales (v.g. el dique "El Cadillal"), sin que se hayan llevado a cabo esfuerzos específicos en el reconocimiento de áreas asociables a la primera mitad del segundo milenio de la Era. Las dificultades inherentes a la ausencia de referencias clásicas sobre yacimientos en la zona, la baja visibilidad superficial, las complicadas condiciones de acceso y permanencia en algunas áreas, así como la reproducción de presupuestos teóricos sobre la marginalidad de las tierras bajas, explican, no sólo el desinterés por generar corpus de herramientas metodológicas nuevas para las tareas de prospección y relevamiento, sino también la consecuente baja proporción de proyectos arqueológicos en el área, en comparación con zonas aledañas (valle de Yocavil, valle de Tafí)

Lo mencionado anteriormente contribuyó a una visión asimétrica de la historia de las sociedades del período en las narrativas arqueológicas argentinas, concentrando los esfuerzos científicos en ciertas áreas de estudio en la región y en elaborar hipótesis a partir de los restos materiales de una acotada fracción del espacio utilizado en el pasado, considerando que éstos son representativos del conjunto (Nielsen et al. 2000). La complejidad de la construcción del paisaje, de las lógicas de uso del espacio y de la configuración de redes de interrelación se ve sesgada por la ausencia de visiones que integren una multiplicidad de datos del registro arqueológico, limitado al análisis exhaustivo de áreas densamente pobladas.

En esta línea, consideramos provechosa la puesta en práctica de distintos procedimientos metodológicos que permitan aumentar los datos sobre las ocupaciones del PDR en la vertiente oriental de las Cumbres Calchaquíes intentando sortear las dificultades intrínsecas a su estudio. En gran medida, si bien las técnicas usuales de identificación de sitios resultan exitosas y han demostrado ser de gran utilidad en el desarrollo del conocimiento arqueológico, la combinación y el refinamiento de las mismas pueden aportar nuevos resultados y de esta manera, contribuir a generar visiones más completas de la distribución de las formas arqueológicas en el paisaje.

En este trabajo se caracterizan los primeros asentamientos asignables al PDR identificados en una cuenca del faldeo oriental de las Cumbres Calchaquíes, Anfama (Dpto. Tafí Viejo, Tucumán), en la cual se implementaron diferentes estrategias para la identificación de sitios. La combinación de distintas metodologías permitió obtener datos de una zona prácticamente desconocida para la arqueología del Noroeste argentino a partir de los cuales se espera contribuir a las reflexiones sobre la naturaleza de las ocupaciones del período en el oriente de las Cumbres Calchaquíes y su relación con los centros poblados del valle de Yocavil.

Este aporte se enmarca en un proyecto más amplio, en el cual se pretenden reconocer las distintas modalidades en las cuales se construyeron y habitaron paisajes a lo largo del tiempo en la cuenca de Anfama. Se considera que estos escenarios son constructos relacionales en los que intervienen agentes humanos y no humanos, los cuales incluyen el entorno físico, sus rugosidades y dinámicas, los ámbitos construidos, la cultura material y la memoria y la percepción de distintos grupos y actores (Anschuetz et al 2001; Criado Boado 1999; Robb 2013). Por lo tanto, el interés consiste en obtener los datos que permitan visualizar en la larga duración cómo diferentes ocupaciones humanas se han sucedido en el paisaje, a modo de capas de un palimpsesto en la materialización de la vivencia y la experiencia de los agentes (Bailey 2007; Lucas 2005), permitiendo la reproducción de prácticas, percepciones y relaciones (Robb 2013).

\section{Área de estudio}

Este trabajo se concentra en el piedemonte oriental de las Cumbres Calchaquíes, en el piso más elevado de la ecoregión de las Yungas: el bosque montano (Cabrera 1976; Oyarzabal et al. 2018). El valle de Anfama se emplaza en la vertiente oriental de las Cumbres Calchaquíes, en el departamento de Tafí Viejo, al Noroeste 
de la provincia de Tucumán. Posee una altitud que varía entre los 1300 y 3000 msnm.

Esta franja altitudinal, lindante con los pastizales de neblina hacia arriba y con la selva montana hacia abajo, muestra la mayor heterogeneidad estructural dentro de las yungas. De esta manera, la topografía es muy escarpada, combinando quebradas muy profundas, con estrechas zonas de cumbres las cuales son levemente planas y dan asiento a la mayoría de las estructuras arqueológicas identificadas. En algunos sectores, especialmente en aquellos próximos a los fondos de la cuenca, se extienden terrenos de menores pendientes con mayor depositación de sedimentos, en donde también se han hallado evidencias de ocupaciones prehispánicas.

En términos generales, el área presenta una vegetación variada, ya que las diferencias en altura, relieve y exposición determinan grandes variaciones climáticas y, por ende, la aparición de diferentes formaciones vegetales. El valle de Anfama se caracteriza por una cobertura vegetal densa, constituida por bosques caducifolios, de coníferas y praderas, por lo cual se pueden encontrar ejemplares de pinos (Podocarpus parlatorei), alisos (Alnus acuminata) y queñoa (Polylepis australis) (Cabrera 1976). La fauna también presenta una alta diversidad, encontrándose diferentes especies de aves, mamíferos y reptiles.

Dentro de la literatura académica, las yungas del NOA han sido poco estudiadas, sólo pudiéndose mencionar algunas excepciones, en su mayoría recientes (Berberián y Soria 1972; Berberián et al. 1977; Caria 2004; Caria y Gómez Augier 2015; Corbalán 2005; Esparrica 1999, 2001; Franco Salvi y Molar 2018; Heredia 1974; Ortiz 2015; Ortiz et al. 2015; Manasse 2011; Núñez y Tartusi 1987; Ventura 1985; Ventura et al. 2010, entre otros). Inclusive, para lo que concierne al bosque montano, no se han realizado hasta el momento investigaciones sistemáticas. En esta línea, la localidad de Anfama constituye un área prácticamente desconocida para la Arqueología argentina, ya que los estudios han sido muy escasos. Solo se tiene registro de dos incursiones expeditivas al área antes del comienzo de los trabajos específicos realizados por el Equipo de Arqueología del Sur de las Cumbres Calchaquíes.

Quiroga (1899), en su paso desde la estancia de La Hoyada hasta la estancia de La Ciénega, menciona la existencia de una serie de estructuras arquitectónicas cuadrangulares con una mala conservación, así como otros recintos ovales, asociados a dos "menhires", y algunas tallas líticas zoomorfas, sin dar cuenta de recolecciones o hallazgos de otros materiales. La descripción de la existencia de recintos rectangulares realizada por el autor, induce a pensar que se trata de un patrón de asentamiento tardío, que contrasta con las unidades circulares propias de momentos tempranos del valle de Tafí (Berberián y Nielsen 1988). Sin embargo, la ausencia de referencias adicionales sobre otros elementos de la cultura material dificulta establecer correspondencias fehacientes sobre la adscripción temporal de las estructuras.

Posteriormente, en los años ochenta, Cremonte (1996) llevó a cabo una serie de sondeos exploratorios con fines comparativos en el marco de su tesis doctoral sobre el asentamiento de La Ciénega (Dpto. Tafí del Valle). De esta manera, identifica en Anfama el sitio Las Cañaditas, cuya arquitectura de planta ortogonal con bloques planos o de lajas alineadas la induce a pensar en la correspondencia de la ocupación con las estructuras mencionadas por Quiroga $\mathrm{y}$, por ende, propone su adscripción a un momento prehispánico tardío. Las diferencias en la composición de las pastas cerámicas y en su manufactura (con la incorporación de tratamientos de superficie como el alisado por marleado) también le permiten sostener esta inferencia. Por otro lado, la baja densidad de materiales culturales recuperados es visualizada por Cremonte (1996) como un indicio del carácter esporádico del asentamiento.

\section{Estrategias para la identificación de sitios del PDR en Anfama}

La cuenca de Anfama muestra una gran cantidad de sitios arqueológicos dispersos en casi todos los puntos habitables del paisaje. Hasta la fecha, el Equipo de Arqueología del Sur de las Cumbres Calchaquíes ha llevado a cabo trabajos sistemáticos de relevamiento, los cuales permitieron identificar 160 estructuras que se agrupan en 10 áreas de concentración de evidencias que denominamos sitios (Salazar et al. 2019). En su mayoría se trata de unidades residenciales construidas, habitadas y abandonadas durante el primer milenio de la Era, inferencia que se sustenta tanto en la observación superficial de sus rasgos arquitectónicos como en los resultados de relevamientos, sondeos, excavaciones en área y fechados radiocarbónicos.

En contraste, los datos sobre ocupaciones correspondientes al PDR eran escasos. No se habían desarrollado estudios sistemáticos sobre la primera mitad del segundo milenio, aunque se tenía evidencia de restos arqueológicos adscribibles de manera estilística a este momento en manos de los comuneros locales, así como la presencia superficial de alfarería de estilos regionales (Santamariano Bicolor y Tricolor, Famabalasto negro grabado, Ordinario peinado) sobre los cortes de terreno o en bloques de adobe de las viviendas actuales. Sin embargo, no existía registro de estructuras arquitectónicas que pudieran ser asignables al período, lo cual llevó a inferir la posible existencia de asentamientos en la zona con un bajo grado de visibilidad o de conservación.

Con el fin de complementar y complejizar el mapa arqueológico de la cuenca, se implementaron una serie de estrategias metodológicas para la identificación de posibles áreas de actividad humana tardías. Este objetivo 
requirió investigar sobre formas alternativas de recolección de datos, así como definir los contextos más apropiados para la aplicación de cada una de ellas (Nielsen et al. 2000). Las actividades desarrolladas conjugaron tanto el trabajo con miembros de la comunidad como la intervención arqueológica, incluyendo la recolección de testimonios orales a los habitantes, el registro de piezas en su poder, prospecciones pedestres, relevamientos de brújula y cinta métrica corregidos a través de imágenes aéreas capturadas por drones y sondeos exploratorios y expeditivos.

\section{Recopilación de datos de comuneros de Anfama}

Bajo la premisa de que los habitantes de la comunidad poseen un conocimiento detallado del territorio y de algunos puntos específicos en los cuales se recuperan frecuentemente objetos arqueológicos se recopilaron datos orales y registraron piezas arqueológicas que los comuneros conservaban en su poder.

En sucesivas entrevistas y charlas informales, en las cuales nuestros informantes proveían datos sobre localizaciones de asentamientos de los "ancestros", se puso énfasis en la detección de algunos rasgos fácilmente identificables y distintivos del PDR, específicamente de cerámicas pintadas y de recintos rectangulares. Estos dos elementos son indicadores cronológicos relativamente confiables, en tanto en la secuencia agroalfarera local solo se registran estilos cerámicos con decoraciones pintadas exclusivamente para la primera mitad del segundo milenio de la Era (i.e. Santamariano bicolor y tricolor y Yocavil polícromo), mientras que previamente se presentan sólo decoraciones incisas y modeladas (Franco, 2018). Por su parte, si bien la existencia de recintos ("corralitos") rectangulares puede resultar menos determinante, esta morfología de plantas de estructuras no se observa en ocupaciones del primer milenio en las cuales predominan las circulares, subcirculares y elípticas (Salazar et al 2019). Complementariamente, en los casos en que los comuneros conservaban piezas arqueológicas también se registraron y fotografiaron considerando especialmente aquellas cuyos estilos eran cronológicamente diagnósticos.

Prospecciones pedestres, relevamientos planimétricos y registro digital

Un segundo grupo de tácticas estuvo dirigida a la identificación, reconocimiento y caracterización de los emplazamientos que puedan albergar asentamientos (Nielsen et al. 2000). Con el objetivo de identificar de manera integral las diferentes ocupaciones prehispánicas en Anfama, no solamente aquellas referidas al PDR, se realizaron prospecciones a pie en la cuenca, ya que los procesos sedimentarios y la densa vegetación del área hizo casi imposible identificar y obtener información sobre sitios mediante técnicas de teledetección.

A causa de la topografía local, caracterizada por desniveles muy pronunciados y quebradas profundas, y de la densa cobertura vegetal que impidieron trazar transectas lineales, o realizar rastrillajes a través de múltiples prospectores (Scheinson, 2001), se utilizaron los ejes que fueron posibles. Muchos de ellos fueron las sendas de pastores que recorren los filos y zonas cumbrales donde a su vez se nuclean la mayoría de los sitios. Desde estos ejes, en los espacios donde fue posible, se cubrieron áreas más amplias. En ningún caso pudieron realizarse recolecciones superficiales debido a la ausencia de materiales arqueológicos en superficie, lo que también constituyó una limitación para la asignación cronológica tentativa de las ocupaciones. La unidad de registro fueron los recintos considerados individualmente, los cuales recibieron un número de ID, consecutivo para todo el proyecto, y sus características fueron descritas en una ficha modelo unificada.

Para la confección de planimetrías de las estructuras, se realizaron relevamientos utilizando cinta métrica, brújula y GPS. La implementación de esta técnica posibilitó la inspección y registro de formas y orientaciones generales de los rasgos, materiales y técnicas constructivas, así como su georreferencia. Sin embargo, en varios casos, las actividades de relevamiento fueron dificultadas por la densa vegetación del área, que genera un grado de visibilidad muy baja de los rasgos arquitectónicos y sobre todo limita la intervisibilidad y el tránsito entre distintos puntos de un mismo sitio, condiciones básicas para el uso de instrumentos de medición topográfica.

Debido a esto se procedió a complementar este tipo de datos con relevamientos aéreos mediante el uso de drone, con el objetivo de definir y mapear con más precisión las estructuras arqueológicas (Thomas y Kennedy, 2016). A través de esta técnica solo fue necesario desmontar de vegetación espacios puntuales que liberaran los vértices de las estructuras y los hicieran visibles en las fotografías cenitales. Estos espacios fueron destacados mediante puntos de color fosforescentes y definidos en un croquis. Las imágenes recogidas fueron gestionadas mediante el programa especializado en fotogrametría digital, Agisoft Photoscan v.1.0.4, para generar ortofotografías georreferenciadas (Sampietro y Pena, 2018); estas últimas, tanto como los croquis obtenidos durante los relevamientos pedestres, fueron exportadas a AutoCad 2016 para realizar la planimetría digital.

Finalmente, toda la información fue sistematizada en QGIS v2.18.21 cuya base fueron las curvas de nivel de los diferentes sitios, obtenidos por el procesamiento de imágenes satelitales con el programa Global Mapper v.16.1.

\section{Sondeos Expeditivos}

En algunos puntos específicos de la cuenca, especialmente en terrazas fluviales de fondos de valle, donde gruesos depósitos sedimentarios cubren en su totalidad a los sitios arqueológicos y la vegetación no permite la exposición de materiales en superficie, realizamos sondeos expeditivos muestreando superficies que 
pueden contener ocupaciones soterradas. Estas intervenciones de muestra son pequeñas cuadrículas de $0.50 \mathrm{~m}$. x $0.50 \mathrm{~m}$. y se distribuyen en dichas terrazas de manera aleatoria con el objetivo de cubrir la mayor extensión posible del área. Estas cuadrículas se excavan de manera expeditiva, en su mayoría utilizando pala, todos los sedimentos son zarandeados y se registra gráficamente la superposición estratigráfica expuesta en los perfiles (Roskams, 2007).

Si bien esta actividad implica la alteración del terreno y de vestigios arqueológicos que pueden no corresponder con los atributos esperados, y puede generar muestras no representativas (Shott, 1989), aplicadas en combinación con otras herramientas tienen la ventaja de ofrecer un conocimiento rápido de las características estratigráficas locales, evidenciar la presencia de restos de actividades humanas donde estos no son visibles en superficie y generar una muestra de materiales que constituyan indicadores cronológicos (Roskams, 2007). Además, permiten definir la posible extensión de espacios de actividad humana que no son visibles en superficie o que no poseen rasgos arquitectónicos, aunque sean áreas que hayan sido utilizadas en el pasado para la realización de algún tipo de tarea.

Esta estrategia también resulta factible de ser complementada con otras actividades, como la recopilación oral de datos, en tanto muchos de los lugares de procedencia de los hallazgos arqueológicos efectuados por los pobladores locales no presentan rasgos en superficie o han sido fuertemente alterados por intervenciones antrópicas subactuales que han afectado las ocupaciones previas (construcción de viviendas, demarcaciones de senderos, actividades agropecuarias, etc.).

\section{Sondeos Exploratorios.}

Después de haber identificado las estructuras y de asignarles cronologías tentativas, definimos una muestra de áreas a ser sondeadas. Los muestreos se realizaron cuadriculando dichos espacios y excavando cuadrículas seleccionadas de manera azarosa. En ellas la excavación siguió estratos naturales, cuyas relaciones estratigráficas fueron registradas a través del método propuesto por Harris (1989). La planificación de cuadriculados permite que los resultados obtenidos en los sondeos se incorporen en excavaciones en área que pueden realizarse en el futuro. Los materiales y rasgos identificados son mapeados y registrados según su posición tridimensional a fin de reconocer concentraciones diferenciales de artefactos. Los perfiles expuestos son registrados gráfica y fotográficamente. Las muestras de vegetales carbonizados a ser fechadas fueron obtenidas en estas intervenciones.

\section{Resultados}

La aplicación de estas cuatro estrategias fue versátil y respondió a las características de cada ocupación, combinados entre sí considerando los resultados que se iban obteniendo. Esto dio por resultado la identificación de 16 estructuras con signos claros de cronología tardía, las que se agrupan en tres sitios.

\section{Casa Rudi 1}

En las inmediaciones de las viviendas de las familias de Rudecindo y Adolfo Chocobar se registraron distintos indicios de actividad humana pretérita, entre los cuales se destacan numerosas superficies de molienda múltiples y fijas próximas al río y materiales arqueológicos, especialmente alfarería, visibles en los cortes de terreno realizados para nivelar la construcción de las casas, así como en los adobes de los muros. Por otro lado, los integrantes de la familia poseían en su poder distintas piezas, como cerámica Santamariana, dos cabezales de hachas, un instrumento de molienda pasivo (molino plano) y una pequeña talla acanalada en uno de sus extremos, formando un motivo fálico (Vázquez Fiorani y Salazar 2018).

En las entrevistas se recuperó información sobre dos sectores A y B del predio familiar en donde se habían extraído en el pasado "tinajones grandes y pucos con dibujos pintados lineales", una descripción que hizo pensar en la posible correspondencia de éstos con piezas de estilo Santamariano.

Los testimonios recuperados fueron contrastados con un sondeo exploratorio (Sondeo Horno) y una excavación en área en las zonas señaladas. A partir de estas intervenciones se definió el sitio Casa Rudi 1 (CR1), en donde se realizó una intervención de grandes dimensiones que permitió identificar un contexto doméstico relacionado a la producción y consumo de alimentos (Vázquez Fiorani y Salazar 2018) y una de menores dimensiones destinada a reconocer la extensión de la ocupación.

En el sector NO del predio de la familia Chocobar se planteó un sondeo de $1 \mathrm{~m} \times 1 \mathrm{~m}$ (Sector B, Sondeo Horno) próximo a un afloramiento granítico ya que allí se habían encontrado materiales arqueológicos según testimonios recogidos en las entrevistas a los dueños de la casa. Se identificaron tres capas superpuestas: UE 501, con mucho material orgánico, que no evidenció material arqueológico y que llegó a los 17,5cm desde la superficie, UE 502, donde se registró un fragmento Famabalasto negro grabado y varios Santamariano bicolor, de $22 \mathrm{~cm}$ de espesor y UE 503 con una potencia de $23 \mathrm{~cm}$, donde se recuperaron una serie de fragmentos de urna Santamariana bicolor de considerable tamaño (Tabla 1). Estos últimos estratos también contaban con presencia de desechos líticos de talla.

En términos generales, el conjunto material presenta características estilísticas tardías, con alfarería tanto decorada como ordinaria. Su correspondencia temporal fue corroborada con un fechado radiocarbónico de un fruto de chañar (Geoffroea decorticans) carbonizado en 
la UE 503, que fue datado en $460 \pm 20$ AP, AP \pm 20 (D-AMS 022989) lo que equivale cal $1421-1452$ cal d.C.

Por su parte, la excavación en área permitió exponer una estructura compuesta por un muro rectilíneo de rocas alineadas horizontalmente, que se caracteriza por su terminación irregular (Vazquez Fiorani y Salazar 2018). El conjunto artefactual permitió corroborar las inferencias realizadas en los sondeos exploratorios, recuperando abundante cerámica de estilos regionales del PDR (Santamariano Bicolor y Tricolor, Famabalasto negro grabado, Ordinario peinado) así como realizar un segundo fechado radiocarbónico: en $465 \pm 20$ AP, $A P \pm 20$ (D-AMS 022988: madera carbonizada) lo que equivale cal 1420- 1450 cal d.C (Vazquez Fiorani y Salazar 2018). Además, se recuperaron desechos de talla e instrumentos líticos (manos de moler, cuchillos, núcleos y otros artefactos formatizados como percutores) de cuarzo, cuarcita roja y en menor medida, obsidiana. Finalmente, se recuperaron restos arqueofaunísticos con marcas de corte antrópico, que fueron identificados como pertenecientes a las familias Lama Sp. y Artiodactyla y que corresponden a los miembros con mayor aporte cárnico (fémur, tibia) (Vázquez Fiorani 2019). Por debajo del piso de ocupación correspondiente a la primera mitad del segundo milenio de la Era, se identificaron restos de materiales arqueológicos asignables a un momento previo. Se trata de un conjunto de fragmentos cerámicos ordinarios, de pasta gruesa oxidante $(n=145)$ similares a los del primer milenio de la Era en la zona (Franco 2019), una concreción de arcilla termoalterada en una de sus caras y abundante carbón vegetal.

\section{Casa Rudi 2}

En la terraza que se extiende en la parte trasera de la vivienda de la familia, se procedió a plantear diez sondeos expeditivos de $0.50 \mathrm{~m}$. x $0.50 \mathrm{~m}$. distribuidos de manera aleatoria dirigida, para cubrir la mayor extensión posible del área $\left(2,5 \mathrm{~m}^{2}\right)$ con el objetivo de identificar indicios de actividad humana prehispánica (rasgos arquitectónicos, cerámica, lítico, etc.) y también definir la extensión de la ocupación donde no se presentan materiales superficiales. Los sondeos fueron denominados calicatas de muestra subsuperficiales (CMS) y distinguidos de manera alfabética consecutiva (i.e CMS-A).

En los diez sondeos de muestra se identificó una estratificación análoga compuesta por una capa superficial de unos 15 a $20 \mathrm{~cm}$ de profundidad (UE 001), un estrato de espesor variable $(20-50 \mathrm{~cm})$, con una gran cantidad de material orgánico y materiales arqueológicos (UE 002 y UE 002b) y un estrato rojizo muy compacto que forma la base de la ocupación antrópica y no permitió reconocer materiales arqueológicos (UE 003) (Figura 1).

De estas intervenciones se extrajo un conjunto material compuesto por desechos de talla lítica y fragmentos cerámicos de distintos estilos claramente asignables al PDR: Santamariano Bicolor, Yocavil polícromo, Ordinario, Ordinario alisado por marleado, así como otros no identificados hasta el momento. En términos estadísticos el material cerámico repite las tendencias ya esbozadas en las excavaciones anteriores con una predominancia de tiestos ordinarios, seguidos por alfarería Santamariana y en menor medida, otros estilos regionales como Famabalasto o Santa María/Belén, aunque con un notable grado de fragmentación.

Esta actividad permitió complejizar la visión sobre la ocupación del sitio Casa Rudi, haciendo inferir una mayor extensión ocupacional en base a la dispersión de elementos de la cultura material no visibles en primera instancia, debido a la alta depositación fluvial del área. En esta línea, pese a la fragmentación del conjunto, la alta densidad de materiales recuperados, así como su dispersión homogénea en el área permite inferir fehacientemente una mayor extensión del asentamiento lo cual no podría haber sido realizado mediante técnicas de identificación superficial. Por otro lado, también permitió apuntar una mayor persistencia en la ocupación temporal del área, ya que los restos tardíos se encuentran dispersos de manera homogénea por la terraza aluvial.

a
\begin{tabular}{|c|r|r|r|r|}
\hline Estilo & UE 502 & \multicolumn{1}{|c|}{ UE 503} & \multicolumn{1}{|c|}{ Total } & \multicolumn{1}{c|}{$\%$} \\
\hline Santamariano Bicolor & & 5 & 5 & 14,71 \\
\hline Santamariano no identificado & 1 & 2 & 3 & 8,82 \\
\hline Famabalasto negro grabado & 1 & 2 & 3 & 8,82 \\
\hline Ordinario & 7 & 5 & 12 & 35,29 \\
\hline Ordinario alisado por marleado & 6 & 5 & 11 & 32,35 \\
\hline
\end{tabular}

$$
\text { b }
$$

\begin{tabular}{|c|c|c|c|c|}
\hline Materia prima & UE 502 & UE 503 & Total & $\%$ \\
\hline Cuarzo & 6 & 8 & 14 & 93,33 \\
\hline Cuarcita roja & - & 1 & 1 & 6,67 \\
\hline Total & 6 & 9 & 15 & 100 \\
\hline
\end{tabular}

Tabla 1. a) Fragmentos ceramicos clasificados segun estilo ceramico y b) Desechos de talla por materia prima del sitio Casa Rudi 1, sector B. c) Fragmentos ceramicos recuperados en Casa Rudi 2 clasificados segun estilo ceramico.

Table 1. a) Ceramic fragments classified according to pottery style. b) Knapped lithic waste by raw material from the Casa Rudi 1 site, sector B. c) Sherds recovered at Casa Rudi 2 classified according to pottery style.

\begin{tabular}{|c|c|c|c|c|c|c|c|c|c|c|c|c|c|}
\hline \multicolumn{14}{|l|}{ c } \\
\hline Estilo & CMS-A & CMS-B & CMS-C & CMS-D & CMS-E & CMS-F & CMS-G & CMS-H & \begin{tabular}{|l|} 
CMS-H UE \\
01 \\
\end{tabular} & CMS-I & CMS-J & Total & $\%$ \\
\hline Santamariano Bicolor & - & 2 & - & 3 & - & - & 2 & - & 4 & 4 & - & 15 & 6,07 \\
\hline Santamariano no identificado & 1 & 4 & 2 & 4 & 1 & - & 1 & 1 & - & 4 & 1 & 19 & 7,69 \\
\hline Famabalasto no identificado & - & 2 & - & 1 & - & - & - & - & - & - & - & 3 & 1,21 \\
\hline Negro sobre rojo & 1 & 5 & - & - & - & - & - & - & - & 1 & - & 7 & 2,83 \\
\hline Yocavil Policromo & 1 & - & - & - & - & - & - & - & - & - & - & 1 & 0,4 \\
\hline Ordinario & 7 & 14 & 7 & 14 & 7 & 6 & 25 & 5 & 15 & 14 & 9 & 123 & 49,8 \\
\hline Ordinario alisado por marleado & 1 & 16 & 2 & 12 & 5 & 3 & 11 & 3 & 3 & 20 & - & 76 & 30,77 \\
\hline No identificado & - & 1 & - & - & - & - & 2 & - & - & - & - & 3 & 1,21 \\
\hline Total & 11 & 44 & 11 & 34 & 13 & 9 & 41 & 9 & 22 & 43 & 10 & 247 & 100 \\
\hline
\end{tabular}



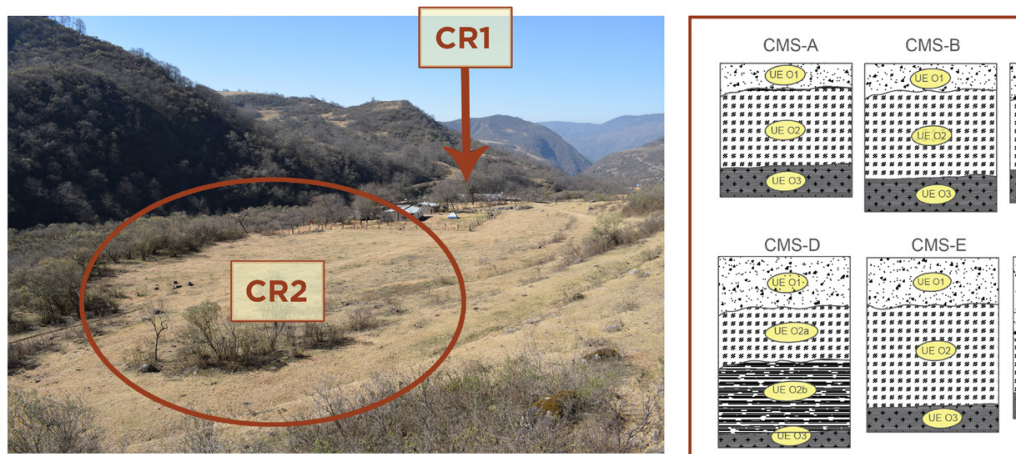

CMS-C
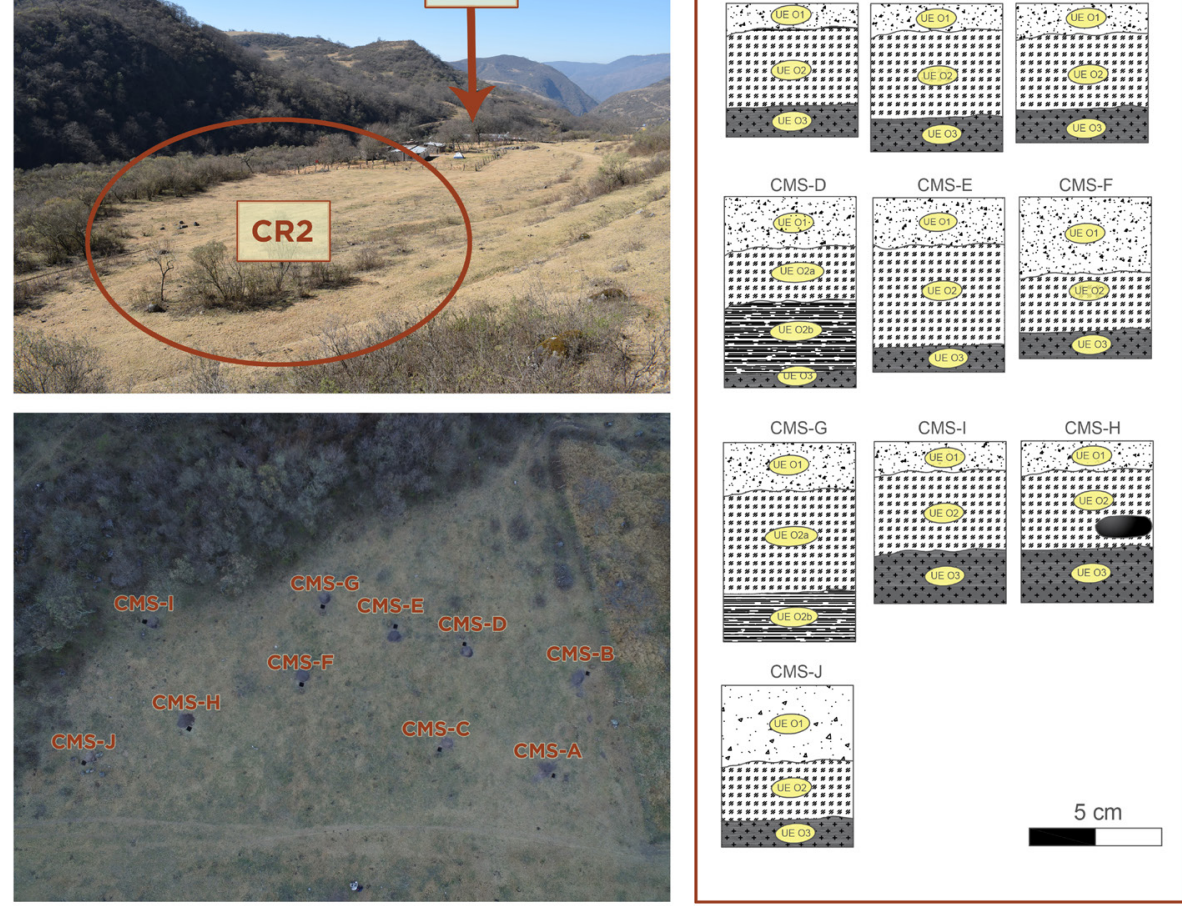

Figura 1. Vista lateral del sitio CR1 y CR2 y detalle de los sondeos expeditivos (izq.). Perfil estratigrafico de las intervenciones realizadas en CR2 (der.).

Figure 1. Lateral view of the CR1 and CR2 sites and detail of the expeditive pit tests (left). Stratigraphy of the interventions carried out in CR2 (right).
Otro resultado positivo fue la identificación de un contexto compuesto por un pequeño rasgo arquitectónico y abundante carbón vegetal en CMS-H. Es importante destacar que este hallazgo nos abre la puerta a nuevas excavaciones en área en contextos que no habrían sido identificables por medio de la observación superficial. Este tipo de estrategias expeditivas permiten aumentar el corpus de información arqueológica y superar los sesgos analíticos presentes. En adición, también permiten considerar prácticas cuya materialización sucede independientemente de los rasgos arquitectónicos, un sesgo importante en la arqueología sobre las sociedades agroalfareras del Noroeste Argentino.

\section{La Laguna}

Como parte del relevamiento general en la cuenca de Anfama, se registraron una serie de estructuras rectangulares emplazadas en lo alto de un cerro, a lo largo del filo denominado La Laguna siguiendo la dirección Noroeste-Sudeste. Este sitio fue destacado por una de las comuneras locales, quien recordaba haber observado allí "tinajas grandes dibujadas". El mismo se halla en un sector neurálgico e ineludible del camino que conecta el valle de Anfama con el valle de Tafí a través de La Ciénega, además de poseer una vista estratégica de las terrazas fluviales de la cuenca. Las características generales observadas guardan una estrecha correlación con un conjunto de estructuras rectangulares mencionadas por Quiroga (Quiroga 1899).

Habiendo identificado la ocupación se realizó un relevamiento planimétrico de las estructuras arqueológicas con brújula y cinta métrica, el cual fue complementado por una ortofotografía generada a partir del procesamiento de 56 fotos capturadas por drone. A través de esto se pudieron delimitar tres unidades arquitectónicas compuestas y de planta cuadrangular (E115, E116, E117, E118; E119, E120 y E250), cuatro simples (E121; E121 y E251) y dos muros de contención (E123 y E124).

La realización de excavaciones en dos unidades permitió obtener datos sobre la técnica constructiva utilizada en las estructuras. Se reconocieron depresiones que corresponden a recintos de planta rectangular, con muros dobles sin relleno de aproximadamente un metro de ancho. Además, se identificaron estructuras monticulares lineales asociadas a rasgos arquitectónicos informales, con baja inversión de trabajo en su manufactura. Se trata de muros formados por rocas dispuestas de manera horizontal, apoyadas sobre el estrato artificial preparado con pequeñas rocas. En este segundo caso, la depositación se evidenció muy irregular, con un notable desnivel N-S.

En primer lugar, se decidió llevar a cabo una serie de sondeos exploratorios en dos estructuras (E116 y E117) con el objetivo de obtener más datos contextuales sobre la adscripción temporal de la ocupación. Se plantearon cuatro sondeos de manera aleatoria (tres en E116 y uno en E117), tres de los cuales se realizaron en sectores intramuros (sondeo 1, extensión 1 y 2) y uno en un sector extramuros que presenta rocas grabadas (sondeo 3).

En el sondeo 1 de la E116 se identificaron dos unidades estratigráficas: UE 800 que constituye la capa superficial 
con gran cantidad de material orgánico hasta los 55 $\mathrm{cm}$ de profundidad y por debajo de ésta, UE 801 de sedimento ligeramente más arenoso hasta los $70 \mathrm{~cm}$, a partir de donde ya se puede observar la roca madre. Esta cuadrícula fue ampliada $50 \mathrm{~cm}$ (Ext. Sondeo 1) hacia el muro del recinto en dirección Norte para terminar de exponer el rasgo arquitectónico y la técnica constructiva, observándose una estratificación análoga. En tanto en E117 se planteó otro sondeo (Sondeo 2) con características depositacionales similares al anterior, aunque con una menor potencia y mayor cantidad de materia orgánica, detectándose la roca madre a los $0,57 \mathrm{~m}$. Finalmente, el sondeo 3 fue realizado en un sector extramuros asociado a un afloramiento de rocas de tamaño considerable las cuales presentaban numerosas cavidades hemiesféricas y surcos lineales grabados en su superficie. En esta unidad solo se reconocieron bloques rocosos aparentemente de un derrumbe, por lo que fue concluido a los $0,51 \mathrm{~m}$.

Con la intención de ampliar la información observada en los sondeos exploratorios, se realizó una excavación en área en E116 y dos trincheras en E120.

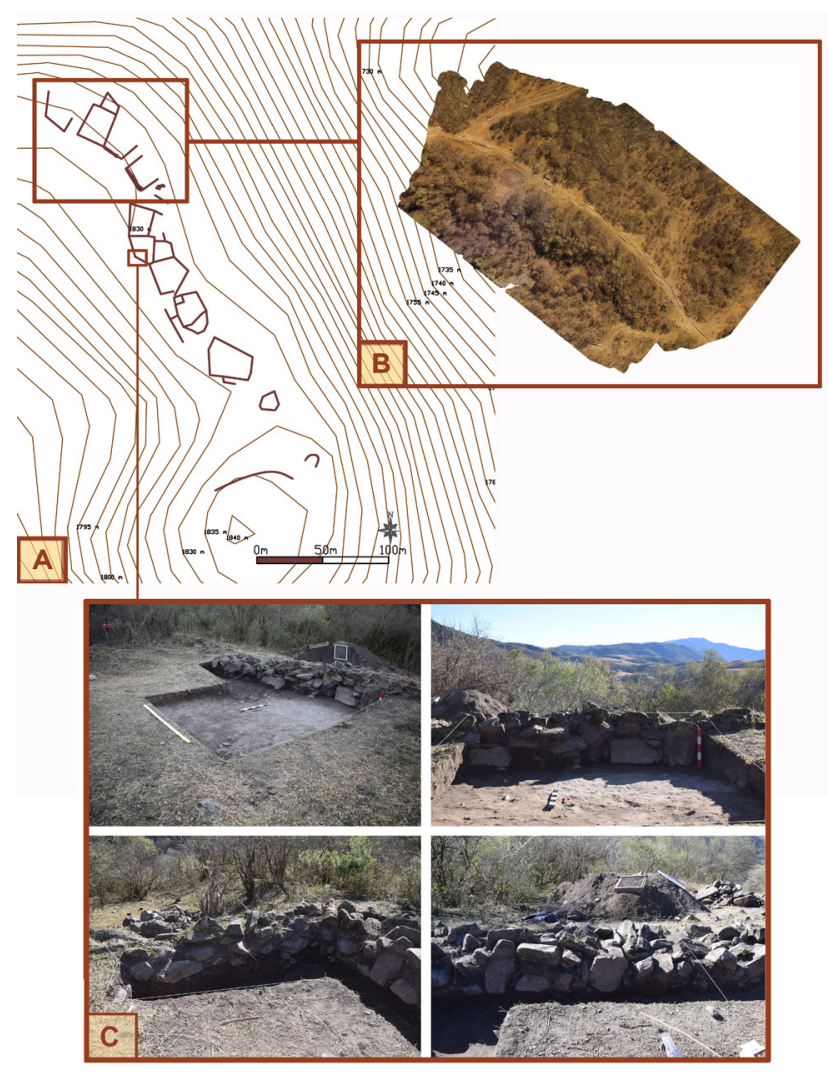

Figura 2. a) Planimetria del sitio La Laguna b) Vista cenital del sitio, donde se puede observar la densa cobertural vegetal c) Detalle de la estructura y excavacion realizada en E116.

Figure 2. a) Archaeological map of the La Laguna site b) Overhead view of the site, where the dense vegetation cover can be observed c) Detail of the structure and excavation carried out in E116.
En el primer caso, se cubrió una superficie de $32 \mathrm{~m}^{2}$ en el extremo Norte del recinto, con características estratigráficas análogas a las observadas en el sondeo original. Entre los estilos representados dentro del conjunto cerámico recuperado de la excavación existe una predominancia de fragmentos ordinarios alisados, así como ordinarios alisados por marleado. En segundo lugar, están representadas el Santamariano y el Famabalasto negro grabado. Entre los restos líticos se pueden mencionar distintos tipos de lascas y desechos de talla, principalmente de cuarzo y cuarcita roja, aunque también se registró obsidiana en menor proporción.

En segundo lugar, se efectuaron dos trincheras en una estructura montícular (E120), una de $5 \mathrm{~m} \times 1 \mathrm{~m}$ en el extremo NE (T01) y otra de $5 \mathrm{~m} \times 2 \mathrm{~m}$ (T02) en el sector centro-oeste de la estructura. Se pudo observar parte de la construcción de la unidad, distinguiéndose en T01 un muro de factura irregular sobre un terreno previamente preparado con pequeñas rocas. La potencia de este sector llegó hasta los $95 \mathrm{~cm}$ de profundidad, exponiéndose un sedimento arcilloso y compacto. A diferencia de esto, T02 se planteó sobre un pequeño rasgo en superficie, de rocas alineadas en forma sub-circular. No se pudo observar la continuación del mismo, que apoyó sobre la roca madre a pocos centímetros de profundidad, aunque con un notable desnivel, sin que la trinchera superara los $50 \mathrm{~cm}$ de potencia. Los restos materiales recuperados en estas intervenciones fueron escasos y repiten la tendencia mencionada en líneas anteriores.

\section{Aliso Redondo-Las Cañaditas.}

En un sector de cumbre se inspeccionó un conjunto de estructuras de difícil adscripción temporal a partir del patrón de asentamiento debido a la superposición de recintos de muros rectilíneos por sobre estructuras circulares. En primer lugar, se efectuó un relevamiento con brújula y cinta métrica que posteriormente fue digitalizado en AutoCad. Se identificaron ocho unidades compuestas (E165, E165 bis; E168; E169, E170; E172, E173; E174, E175; E176, E177 y E178, E179, E180) y dos rasgos arquitectónicos rectilíneos (E181).

Una de las unidades presentaba dos recintos, uno circular (E165) por sobre el cual se observaba la superposición de una estructura rectangular (E165 bis). Además, en las inmediaciones se encontró una piedra grabada con un conjunto de cavidades hemiesféricas dispuesta como parte del muro de la estructura de planta oval. También se registraron instrumentos de molienda fijos y móviles fracturados en superficie. Algunas de las características arquitectónicas de las estructuras (muros de bloques líticos alineados horizontalmente, planta rectangular) hacían pensar en la existencia de ocupaciones tardías superpuestas a las unidades del primer milenio de la Era, por lo cual se efectuaron dos sondeos exploratorios.

Se planteó un sondeo de $1 \mathrm{~m} \times 1 \mathrm{~m}$ en el área central de 
A

\begin{tabular}{|c|c|c|c|c|c|c|c|c|}
\hline \multirow{3}{*}{ Estilo } & \multicolumn{4}{|c|}{ E116 } & \multirow{2}{*}{\multicolumn{2}{|c|}{$\begin{array}{c}\text { E117 } \\
\text { Sondeo } 2\end{array}$}} & \multirow{3}{*}{ Total } & \multirow{3}{*}{$\%$} \\
\hline & \multicolumn{2}{|c|}{ Sondeo 1} & \multicolumn{2}{|c|}{ Extensión Sondeo 1} & & & & \\
\hline & UE 800 & UE 801 & UE 800 & UE 801 & UE 850 & UE 851 & & \\
\hline $\begin{array}{c}\text { Santamariano } \\
\text { Bicolor }\end{array}$ & 2 & & & & & & 2 & 1,11 \\
\hline $\begin{array}{c}\text { Santamariano } \\
\text { no identificado }\end{array}$ & 20 & 3 & & 9 & & 2 & 34 & 18,89 \\
\hline $\begin{array}{l}\text { Negro sobre } \\
\text { rojo }\end{array}$ & & & & 1 & & & 1 & 0,56 \\
\hline $\begin{array}{c}\text { Famabalasto } \\
\text { negro grabado }\end{array}$ & & & & 9 & & 1 & 10 & 5,56 \\
\hline No identificado & & 1 & & & & & 1 & 0,56 \\
\hline Ordinario & 26 & 31 & 3 & 18 & 9 & 7 & 94 & 52,22 \\
\hline $\begin{array}{l}\text { Ordinario } \\
\text { alisado por } \\
\text { marleado }\end{array}$ & 5 & & & 4 & 4 & 4 & 38 & 21,11 \\
\hline Total & 53 & 35 & 3 & 41 & 13 & 14 & 180 & 100 \\
\hline
\end{tabular}

B

\begin{tabular}{|c|c|c|c|c|c|c|c|c|}
\hline \multirow{3}{*}{ Materia prima } & \multicolumn{4}{|c|}{ E116 } & \multirow{2}{*}{\multicolumn{2}{|c|}{$\begin{array}{c}\text { E117 } \\
\text { Sondeo } 2\end{array}$}} & \multirow{3}{*}{ Total } & \multirow{3}{*}{$\%$} \\
\hline & \multicolumn{2}{|c|}{ Sondeo 1} & \multicolumn{2}{|c|}{ Extensión Sondeo 1} & & & & \\
\hline & UE 800 & UE 801 & UE 800 & UE 801 & UE 850 & UE 851 & & \\
\hline Cuarzo & 12 & 2 & - & 4 & 2 & 1 & 21 & 55,26 \\
\hline Cuarcita roja & 2 & 2 & - & 4 & 2 & - & 10 & 26,32 \\
\hline Cuarcita gris & - & - & - & - & - & - & - & - \\
\hline Obsidiana & - & - & - & - & - & 1 & 1 & 2,63 \\
\hline No identificado & 3 & 2 & - & 1 & - & - & 6 & - \\
\hline Total & 17 & 6 & - & 9 & 4 & 2 & 38 & 100 \\
\hline
\end{tabular}

Tabla 2. a) Fragmentos ceramicos clasificados segun estilo ceramico. b) Desechos de talla por materia prima del sitio La Laguna.

Table 2. a) Sherds classified according to pottery style. b) Knapped lithic waste by raw material from the La Laguna site.
E165bis que posteriormente fue expandido para exponer un posible rasgo, el cual una vez descubierto se interpretó como un derrumbe del muro del recinto.

En la excavación se recuperó un conjunto material discreto, compuesto principalmente por cerámica Santamariana y Ordinaria alisada por marleado así como algunos desechos de talla y artefactos líticos (una mano de moler y un núcleo). Por las características de la materialidad en las capas inferiores, donde desaparecen totalmente los estilos adscritos usualmente al PDR y aumenta la proporción de alfarería sin decoración, muy similar a la del primer milenio de la Era en Anfama (pastas gruesas de cocción oxidante con abundancia de inclusiones expuestas en la superficie), se propone que la ocupación tardía se habría emplazado por encima del asentamiento temprano, como también se vislumbra a partir de la modificación arquitectónica del recinto.

\section{Discusión}

La implementación de las diferentes estrategias metodológicas permitió identificar hasta el momento tres sitios correspondientes al PDR en la cuenca de Anfama. De esta manera, se logró avanzar en la complejización del mapa arqueológico de la zona, evidenciando una densidad de ocupación durante la primera mitad del segundo milenio superior a lo que se pensaba hasta el momento. Por otro lado, también permitió superar algunas de las dificultades inherentes al terreno, como la baja visibilidad y la escasa conservación superficial de los restos arqueológicos que en gran medida sesgan la muestra obtenida sobre las lógicas de uso del espacio.
Las ocupaciones del PDR en la cuenca de Anfama identificadas hasta el momento evidencian la adopción de pautas arquitectónicas de difusión regional, asociadas al valle de Yocavil. De esta manera, se caracterizan por estructuras de muros rectilíneos de rocas dispuestas de manera horizontal, aunque emplazadas en espacios relativamente abiertos sin restricciones para su acceso o circulación. Sin embargo, un elemento que se desprende del análisis aquí presentado es la tendencia a reocupar espacios que ya habían estado en uso durante el primer milenio de la Era, lo que evidencia una íntima relación (directa o indirecta) entre ambos momentos ocupacionales.

En primer lugar, las ocupaciones de cumbre se encuentran en sectores de pendientes relativamente pronunciadas con muy pocos terrenos horizontales, por lo cual los espacios apropiados para el asentamiento son limitados. En el caso de La Laguna, el sitio presenta una visibilidad muy buena de todo el fondo de valle, pudiéndose observar en un ángulo de $180^{\circ}$ toda la franja adyacente al río de Anfama, en donde también se encontraron distintos indicios de actividad humana prehispánica (CR1, CR2). Por otro lado, desde el mismo punto también se tiene una visión privilegiada del sector Oeste de las Cumbres Calchaquíes pudiéndose controlar el filo que conecta Anfama con La Ciénega. Pese a su ubicación, Aliso Redondo-Las Cañaditas no posee un grado de visibilidad del entorno semejante, aunque se puede acceder a una vista parcial del sector Norte-Este de la cuenca.

En estos asentamientos, los conjuntos arquitectónicos se presentan de manera aislada o compuesta, con grandes muros dobles de rocas dispuestas de manera 


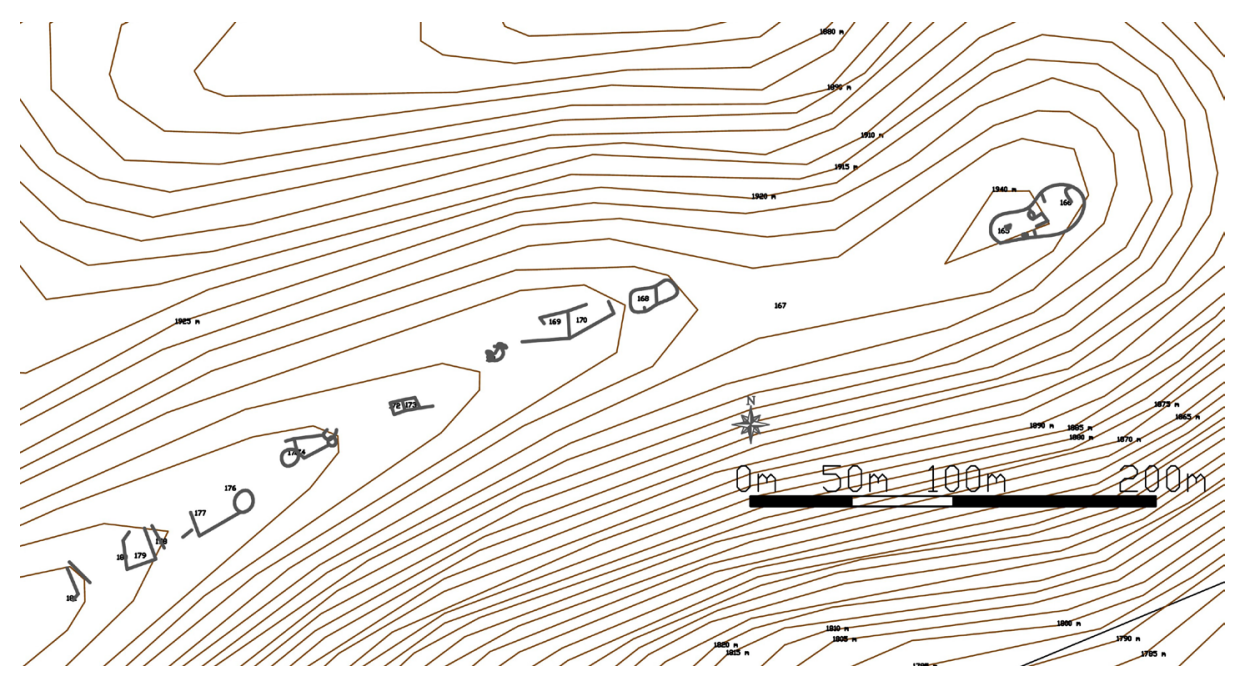

Figura 3. Planimetria sitio Aliso Redondo-Las Cañaditas.

Figure 3. Archaeological map of the Aliso Redondo-Las Cañaditas site.

horizontal. Se trata de una arquitectura formal, que en el caso particular de La Laguna evidencia una alta inversión de tiempo en la construcción de los recintos. La posible reocupación tardía de un sitio formativo en Aliso Redondo-Las Cañaditas parece haber modificado las estructuras circulares tempranas, mediante la superposición de nuevos muros de traza rectangular que literalmente "cortan" la unidad original. Es interesante notar, que este patrón de reocupación ya fue observado en un sitio de fondo del valle de la cuenca, El Sunchal, en donde se superponen al menos dos ocupaciones, una fechada a principios del primer milenio de la Era y otra a fines del mismo (Salazar et al. 2019).

A diferencia de este tipo de ocupaciones, el sitio identificado en el fondo de la terraza fluvial (Casa Rudi) se encuentra en un área abierta, de pendientes suaves y poco pronunciadas. Al encontrarse en un punto del terreno de poca altitud, la visibilidad general es relativamente baja, sobre todo si se tiene en cuenta la profusa vegetación existente. La arquitectura tiene un carácter informal, que, a diferencia de las estructuras más formales de los sectores de cumbre, parece haber requerido una baja inversión de tiempo y en general, forma lienzos irregulares en su terminación. Además, la mayor depositación de sedimentos eólicos y fluviales en estos asentamientos genera que muchos de los rasgos arquitectónicos se encuentren soterrados, por lo cual la visión que tenemos en la actualidad es parcial y limitada.
Sin embargo, cabe mencionar que entre La Laguna y Casa Rudi la visibilidad es privilegiada, encontrándose ambos sitios intrínsecamente vinculados. De esta manera, la distancia entre las ocupaciones podría haber sido sorteada por la conexión visual, lo cual también se observa para las ocupaciones del primer milenio de la Era en el área, una estrategia que permitiría la conformación y reproducción de lazos entre unidades domésticas que pretendían un grado de autonomía (Salazar et al. 2019).

De esta manera, vemos que la construcción del paisaje durante el PDR tiende a repetir algunas lógicas observadas en momentos previos, con unidades residenciales discretas que se emplazan de manera dispersa en el paisaje (Salazar et. al. 2019). Las ocupaciones tardías tienden a emplazarse en espacios utilizados con anterioridad, como se observó en el caso de Casa Rudi y Aliso Redondo-Las Cañaditas, en donde las unidades del PDR se superponen a estructuras previas del primer milenio de la Era.

A título tentativo, podemos postular como hipótesis a trabajar en el futuro que la experiencia de los agentes implicó un contacto íntimo con los resabios de ocupaciones previas, así como también con las prácticas de larga duración que implicó la construcción de un paisaje caracterizado por la dispersión y la intervisibilidad. De esta manera, tanto la materialización de la experiencia diaria de contacto con ese pasado, como la recreación de la memoria del mismo habrían
A

A
\begin{tabular}{|c|c|c|c|c|c|c|}
\hline Estilo & UE 450 & UE 451 & UE 453 & UE 455 & Total & $\%$ \\
\hline Santamariano no identificado & 2 & 10 & - & - & 12 & 19,35 \\
\hline Ordinario & - & 37 & 4 & 3 & 44 & 70,97 \\
\hline Ordinario alisado por marleado & 2 & 4 & - & - & 6 & 9,68 \\
\hline Total & 4 & 51 & 4 & 3 & 62 & 100 \\
\hline
\end{tabular}

$$
\text { B }
$$

B
\begin{tabular}{|c|c|c|c|c|}
\hline Materia prima & UE 450 & UE 451 & Total & $\%$ \\
\hline Cuarzo & 2 & 10 & 12 & 66,67 \\
\hline Cuarcita roja & - & 6 & 6 & 33,33 \\
\hline Total & 2 & 16 & 18 & 100 \\
\hline
\end{tabular}

Tabla 3. a) Fragmentos ceramicos clasificados segun estilo ceramico. b) Desechos de talla por materia prima del sitio Aliso Redondo-Las Cañaditas.

Table 3. a) Sherds classified according to pottery style. b) Knapped lithic waste by raw material from the Aliso Redondo-Las Cañaditas site. 
sido elementos cruciales para la construcción y el habitar de los paisajes en Anfama, pero también para el mantenimiento de pautas y tradiciones locales en un momento marcado por fuertes cambios sociales, políticos y culturales a nivel regional.

\section{Palabras finales}

Como se mencionó, uno de los principales problemas metodológicos a los que se enfrentan las investigaciones sobre el PDR en el piedemonte oriental de las Cumbres Calchaquíes es el que se refiere a la baja visibilidad de los asentamientos. En gran medida, es de suponer que las dificultades para identificar sitios haya sido uno de los condicionantes para el desarrollo sistemático de trabajos en el área, los cuales hasta el momento tienen un carácter excepcional.

En este trabajo, se partió de una premisa tentativa que postuló la posible presencia de ocupaciones asignables al PDR que no fueran visibles en primera instancia sobre la superficie, y que de esta manera, se caracterizaran por su emplazamiento discreto sobre el paisaje, su baja densidad ocupacional o por haber sido afectadas gravemente por la alteración de procesos posdepositacionales naturales y antrópicos. La combinación de las diferentes estrategias se mostró particularmente útil en el caso aquí tratado, en tanto permitió obtener datos originales sobre un área donde no se tenía conocimiento fehaciente de ocupaciones correspondientes a la primera mitad del segundo milenio de la Era. De esta manera, la información obtenida en los distintos contextos de actividad humana (Casa Rudi, La Laguna y Aliso Redondo-Las Cañaditas) proporcionó elementos que pueden aportar y complejizar la discusión sobre la naturaleza de las ocupaciones humanas durante el PDR en un área donde la mayoría de las interpretaciones generales sobre las dinámicas sociopolíticas aún no han sido puestas a prueba sistemáticamente.

Córdoba, octubre de 2019.

\section{Agradecimientos}

Este trabajo fue realizado gracias al apoyo económico de las siguientes instituciones nacionales e internacionales: SECYT (UNC); SPU; CONICET; FONCyT; Koeki Zaidan Hojin Toyota Zaidan (公益財団法人卜ヨ夕財団) The Toyota Foundation [TYTID: D16-R-0718]; National Geographic Society [W464-16]. Además, agradecemos a los miembros de la Comunidad Indígena Diaguita de Anfama y a los colaboradores de campañas y tareas de gabinete 20162018, especialmente a la Dra. Valeria Franco Salvi, Dra. Valeria Palamarczuk y Lic. a Francisco Franco.

\section{Bibliografía}

Anschuetz, K., R. Wilshusen \& C. Scheick. (2001). An Archæology of Landscapes: Perspectives and Directions. Journal of Archoeological Research, 9(2), 152-197. Doi: 10.1023/A:1016621326415
Bailey, G. (2007). Time perspectives, palimpsests and the archaeology of time. Journal of Anthropological Archaeology, 26, 198-223. Doi: 10.1016/j.jaa.2006.08.002

Berberián, E. \& Soria, D. (1972). Investigación arqueológica en el Yacimiento de Zárate (Departamento de TrancasTucumán). Informe preliminar. Humanitas. Revista de la Facultad de Filosofía y Letras, 22, 165-176.

Berberián, E., García Azcarate, J. \& Caillou, M. (1977) Investigaciones arqueológicas en la región del dique El Cadillal (Tucumán, Rep. Argentina). Los primeros fechados radiocarbónicos. Relaciones de la Sociedad Argentina de Antropología 9, 31-53.

Cabrera, A. (1978). Fitogeografía de la República Argentina. Boletín de la Sociedad Argentina de Botánica, 14, 1-42.

Caria, M. \& Gómez Augier, J. (2015). Arqueología en espacios contrastados en los piedemontes oriental y occidental de Cumbres Calchaquíes (Tucumán-Argentina) durante el $1^{\circ}$ y $2^{\circ}$ milenio de nuestra Era. En Crónicas materiales precolombinas. Arqueología de los primeros poblados del Noroeste Argentino (1era edición, pp. 355-383). Disponible en: http:// www.saantropologia.com.ar/wp-content/uploads/2016/01/ CRONICAS-MATERIALES-PRECOLOMBINAS2015.pdf

Corbalán, M. (2008). Periferia y marginalidad en la construcción arqueológica: las sociedades prehispánicas tardías de las estribaciones orientales de las Cumbres Calchaquíes (noroeste de Argentina). Maguaré, 22, 365395. Doi: $10.15446 /$ mag

Cremonte, M.B. (1996). Investigaciones arqueológicas en la Quebrada de La Ciénaga (Dto. Tafí, Tucumán). Tesis doctoral inédita. Universidad Nacional de La Plata.

Criado Boado, F. (1999). Del terreno al espacio: planteamientos y perspectivas para la arqueología del Paisaje. CAPA 6. Grupo de Investigaciones en Arqueología del Paisaje. Santiago de Compostela.

Esparrica, H. (1999). Investigaciones arqueológicas en el sitio S. Tuc. Tra 21, Mortero Hachado, dpto. Trancas, prov. Tucumán. En Actas del XII CNAA, Tomo II: 82-91. La Plata.

Franco Salvi, V., \& Molar, R. M. (2018). Paisajes agrarios del segundo milenio de la era en el sector norte del Valle de Tafí (Tucumán, Argentina). Estudios Atacameños, 57, 45-63. Doi: 10.4067/S0718-10432018005000602

Franco, F. (2019). La práctica alfarera durante el primer milenio de la Era en El Sunchal, Anfama (Dto. Tafí Viejo, Tucumán, Rep. Argentina). Trayectorias de vida, cadenas operativas y ¿tradición?. Tesis de licenciatura inédita. Universidad Nacional de Córdoba.

Harris, E. (1991). Principios de estratigrafía arqueológica (1era edición en español). Barcelona: Crítica.

Heredia, O. (1974). Investigaciones arqueológicas en el 
sector meridional de las Selvas Occidentales. Revista del Instituto de Antropología, 5, 73-132.

Lucas, G. (2005). The archaeology of time (1era. edición). Inglaterra: Routledge.

Manasse, B. (2011). Arqueología en el borde andino del Noroeste argentino. Sociedades del último milenio en el Valle de Tafí, Prov. De Tucumán, República Argentina. Tesis de doctorado inédita. Universidad Nacional de La Plata.

Nastri, J. (1999) Patrones de asentamiento prehispánicos tardíos en el sudoeste del valle de Santa María (noroeste argentino). Relaciones de la Sociedad Argentina de Antropología, 22-23, 247-270.

Nielsen, A., Ávalos, J. \& Menacho, K. (2000). Más allá del sitio: el registro arqueológico de baja densidad y su importancia para el estudio de sociedades agroalfareras. Revista del Museo de La Plata. Nueva Serie, 9(83), 355-370.

Núñez Regueiro, V. \& Tartusi, M. (1987). Aproximación al estudio del área pedemontana de Sudamérica. Cuadernos Instituto Nacional de Antropología, 12, 125-160.

Ortiz, G. (2015). Avances y nuevas perspectivas en la arqueología del piedemonte de Jujuy (Valle de San Francisco), Argentina. En Alconi, S. \& Jaimes Betancourt, C. (Ed.), En el corazón de América del Sur 3. Arqueología de las tierras bajas de Bolivia y zonas limítrofes (pp. 195213). Santa Cruz de la Sierra: Imprenta 2E.

Ortiz, G., Heit Lanart, C., Nieva, L., Zamora, F., Batallanos, N. \& Chapur, F. (2015). Pensando al Formativo desde la región pedemontana de las yungas de Jujuy. En Crónicas materiales precolombinas. Arqueología de los primeros poblados del Noroeste Argentino (1era edición, pp. 695720). Disponible en: http://www.saantropologia.com.ar/ wp-content/uploads/2016/01/CRONICAS-MATERIALESPRECOLOMBINAS2015.pdf

Oyarzabal et al. (2018). Unidades de vegetación de la Argentina. Ecología austral, 28, 40-63. Doi: 10.25260/ EA.18.28.1.0.399

Palamarczuk, V. (2016). Investigaciones arqueológicas en El Colorado, sur de Yocavil, Catamarca, Argentina. Andes, 27(1), 1-28.

Quiroga, A. (1899). Ruinas de Anfama. El pueblo prehistórico de la Ciénega. Boletín del Instituto geográfico argentino, 20, 95-123.

Roskams, S. (2007). Excavation (1era. edición en inglés). Cambridge: Cambridge University Press.

Sabloff, J, \&W. Ashmore (2001). An aspect of archaeology's recent past and its relevance in the New Millenium. En Feinman, G. y Price, D., Archaeology at the millennium (1era edición, pp. 11-32). Nueva York: Springer.

Salazar, J. et. al. (2019) Investigaciones arqueológicas en la cuenca de Anfama, provincia de Tucumán. En A. Laguens, M. Bonnin \& B. Marconetto (comp.), Libro de resúmenes del XX CNAA. Córdoba: IDACOR.

Sampietro, M. M. \& J. Peña (2018). Aplicación de los drones en diversos contextos arqueológicos y en casos de reconstrucción geoarqueológica. Comechingonia 22(1): 271-293

Scheinsohn, V. (2001). Odisea del espacio. Paisajes y distribuciones artefactuales en Arqueología. Resultados y propuestas. Relaciones de la Sociedad Argentina de Antropología 24, 285-201.

Shott, Michael. (1989). Shovel-Test Sampling in Archaeological Survey: Comments on Nance and Ball, and Lightfoot. American Antiquity, 54 (2): 396-404.

Tarragó, M. (1987). Sociedad y sistema de asentamiento en Yocavil. Cuadernos del Instituto Nacional de Antropología, 12, 179-196.

Tarragó, M. (1995). Desarrollo regional en Yokavil: una estrategia de investigación. En Actas XIII Congreso Nacional de Arqueología Chilena. Antofagasta de la Sierra.

Thomas, H. \& M. Kennedy (2016). A new methodology for accurate digital planning of archaeological sites without the aid of surveying equipment. Journal of Archaeological Science, 10, 887-892. Doi: 10.1016/j.jasrep.2016.06.006

Vazquez Fiorani, A. (2019). Revising the Zonal Complementarity model in the Eastern slopes of the Cumbres Calchaquíes: contributions from Household Archaeology (Tucumán, Northwest Argentina). Journal of Anthropology and Archaeology, 7(1), 23-28. Doi: 10.15640/jaa.v7n1a3

Vazquez Fiorani, A. \& Salazar, J. (2018). Nuevos datos sobre ocupaciones tardías en la vertiente oriental de las Cumbres Calchaquíes: el sitio Casa Rudi 1 (Anfama, Provincia de Tucumán). Relaciones de la Sociedad Argentina de Antropología, 43 (2), 287-296.

Ventura, B. (1985). Representaciones de camélidos y textiles en sitios arqueológicos tardíos de las selvas occidentales. Relaciones de la Sociedad Argentina de Antropología, 36, 191-202.

Ventura, B., Delcourt, P., Ortiz, G., Methfessel L., Greco, C., Buitrago, W. y Paredes, F. (2010). El registro arqueológico de las antiguas poblaciones de los valles orientales de la Provincia de Arce, Tarija, Bolivia. Intersecciones en Antropología, 11(1), 59-72. 